\title{
Instrumento para la medición de la temperatura de las personas a distancia
}

\author{
Gianni Pezzotti*\$, Paolo Coppa**, Fabrizio Liberati*** \\ *Departamento de Investigación y Desarrollo, Biosensor SRL e Instituto de Cristalografia, \\ Consiglio Nazionale delle Ricerche "CNR", Roma, Italia \\ **Departamento de Ingeniería Mecánica, Facultad de Ingenieria, \\ Universidad degli Studi di Roma "Tor Vergata", Roma, Italia. \\ ***Departamento de Diseño Optoservice SRL Capagnano, Roma, Italia \\ $\S$ e-mail: g.pezzotti@biosensor.it
}

(Recibido: Abril 12 de 2005 - Aceptado: Julio 11 de 2006)

\section{Resumen}

Se presenta una nueva aplicaciòn basada en un pirómetro a baja radiación para medir la temperatura del cuerpo humano. El instrumento se basa en una termopila de bajo costo como detector y un sistema óptico consistente en un espejo cóncavo elíptico. Fue especialmente diseñado para medir la temperatura de la frente de una persona. El instrumento ha sido caracterizado usando un cuerpo negro construido para este propósito. La precisión del instrumento es de $0.19^{\circ} \mathrm{C}$.

Palabras Clave: Pirómetro de baja radiación, Termopila, Sistema óptico, Temperatura de la piel.

\begin{abstract}
A new low radiation pyrometer to measure the temperature of the human body is presented. The instrument is based on a low cost thermopile as detector and an optical system consisting of an elliptical concave mirror. It was specially designed to measure the temperature of a person's forehead. The instrument has been characterized using a black body built for this purpose. The precision of the instrument is $0.19^{\circ} \mathrm{C}$.
\end{abstract}

Keywords: Low temperature pyrometer, Thermopile, Optical system, Skin temperature.

\section{Introducción}

Una de las primeras aplicaciones de la termometría fue la medición de la temperatura del cuerpo humano. Aún hoy, nuestra idea de la medición de la temperatura se relaciona con el control de la fiebre mediante el uso de termómetros de contacto y mercurio en vidrio. Estos instrumentos son ampliamente utilizados, son seguros, precisos $\left( \pm 0.1^{\circ} \mathrm{C}\right.$ como estándar de incertidumbre) y fáciles de usar. Desafortunadamente, estos termómetros requieren de la inserción del instrumento, y su tiempo de respuesta es largo (3-5 minutos). Esta es la razón por la que en los últimos años han aparecido en el mercado termómetros para oído (i.e, Braun IRT 3520), estos instrumentos son pirómetros de baja temperatura que usan termopilas como detectores y que miden la temperatura interna del oído considerando esta cavidad como un cuerpo negro isotérmico con respecto al interior del cuerpo humano. $\mathrm{Su}$ respuesta es mucho más rápida (cerca de 1 segundo) y su precisión es comparable con los termómetros de vidrio. Estos instrumentos pueden considerarse como no invasivos, pues las mediciones se realizan asomando el instrumento al oído. Sin embargo, algunos tipos de aplicación no pueden realizarse con estos sensores, 
particularmente si las personas están en movimiento, como por ejemplo: el monitoreo continuo de la temperatura corporal de pacientes en cama en los hospitales, la medición de la temperatura de las personas que cruzan las puertas de los aeropuertos (para monitorear y detectar posibles viajeros infectados con virus peligrosos como el ebola, fiebre aviar, etc.), los detectores de movimiento, las alarmas de seguridad, el monitoreo de la temperatura de las personas en ambientes cerrados (salas, vehículos, etc.) para regulaciones climáticas (Taniguchi et al., 1992; Lee et al., 1998). En la figura 1 se observan algunas de las aplicaciones mencionadas.

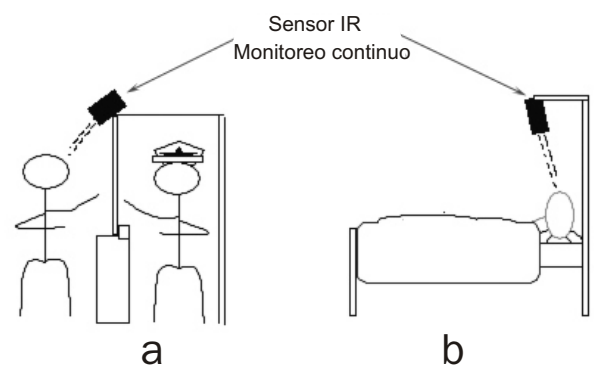

Figura 1. Algunas de las aplicaciones identificadas para un nuevo sistema de medición térmica.

a. Entradas de inmigracion en aeropuertos

b. Sala de cuidados intensivos

La frente es la parte externa del cuerpo humano con temperatura más constante, casi independiente de la temperatura del ambiente (Kataoka et al., 1998), y generalmente es también la parte más fácilmente accesible del cuerpo humano, estando su piel desnuda. Además la piel puede ser considerada como una muy buena aproximación de un cuerpo negro en el rango de longitudes de onda de 1 a $10 \mu \mathrm{m}$ (que incluye el rango de energía infrarroja emitida por los humanos) y las mediciones de temperatura radiante no necesitan corrección por emisividad.

El objetivo inicial de este trabajo consistió en el diseño, construcción, caracterización y prueba de un pirómetro de baja temperatura capaz de medir la temperatura de la frente humana. Se requerían unas características particulares del instrumento como tamaño pequeño, facilidad de uso, bajo costo (por lo menos para producción en masa), fácil construcción y muy buena precisión (cerca a $\left.40^{\circ} \mathrm{C}\right)$.

Los pirómetros de baja temperatura son productos comunes en el mercado (i.e., Minolta-Land Infrared, Ircon, Omega, etc.), pero ellos generalmente usan sensores enfriados por nitrógeno líquido o enfriados termoeléctricamente y lentes de germanio por lo que su costo resulta bastante alto.

Para satisfacer los requerimientos deseados para las aplicaciones descritas, se decidió construir un instrumento con las siguientes características: Dimensiones reducidas ( $60 \mathrm{~mm}$ de ancho y largo), rango de longitudes de onda entre 3 y $15 \mu \mathrm{m}$ para obtener resultados en el rango deseado de temperaturas, entre 30 y $40^{\circ} \mathrm{C}$; la imagen del objetivo es de $2 \mathrm{~cm} \times 2 \mathrm{~cm}$ suficientemente grande para entregar una señal detectable y suficientemente pequeña para garantizar un buen enfoque en la frente, distancia de trabajo entre 350 y $400 \mathrm{~mm}$, de forma de obtener una buena señal cuando se presentan movimentos leves de algunos centimetros. El sistema óptico es diseñado de manera de forma que cualquier movimiento de la persona, no disturbe la señal. En el diagrama de bloques de la figura 2 se describe el funcionamiento del instrumento propuesto.

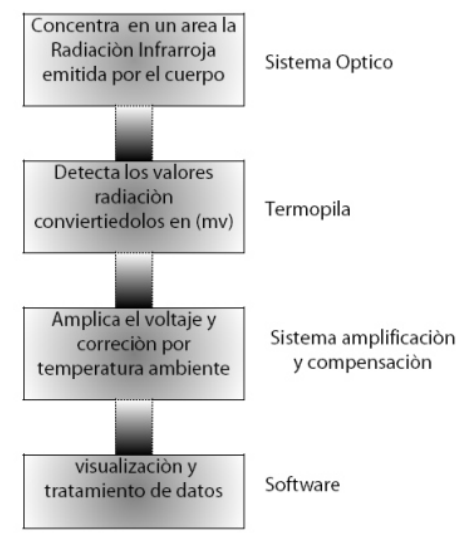

Figura 2. Breve descripción del funcionamiento del instrumento para la medición de la temperatura de la frente.

Este articulo ha sido estructurado de la siguiente manera: en la sección 2.1 se ilustra el sistema óptico, y en la sección 2.2 se presenta el sistema mecánico. En la sección 2.3 se describe el sensor de radiación y en la sección 2.4 se trata la caracterización del instrumento. En la sección 3.2 
se describen las pruebas preliminares y sus resultados. En la sección 3.3 se realiza un análisis sobre la precisión del instrumento y en la sección 4 se presentan las conclusiones y recomendaciones.

\section{Metodologia}

\subsection{Sistema óptico}

El sistema óptico ha sido diseñado para transmitir el máximo flujo radiante hacia el sensor. Este sistema consiste en un solo espejo de forma circular, y que no es plano en la superficie, sino con una concavidad elíptica para minimizar la aberración esférica. Está hecho en aluminio, con la superficie reflectora cubierta de oro para evitar la oxidación y asegurar la máxima reflexión de la radiación en el rango de longitudes de onda correspondiente entre 20 y $45^{\circ} \mathrm{C}$. En este rango de longitudes de onda no se requiere de un acabado demasiado perfecto en la superficie, es suficiente con el que se obtiene con un maquinado de aproximadamente $0.5 \mu \mathrm{m}$. El diseño óptico está basado en los siguientes requerimientos: diámetro del espejo $50 \mathrm{~mm}$, distancia focal $375 \mathrm{~mm}$ y magnificación de 0.1 (para obtener una imagen de $2 \mathrm{~mm} \times 2 \mathrm{~mm}$, igual al área del sensor, de un objetivo de $20 \mathrm{~mm}$ x $20 \mathrm{~mm}$ ). En la figura $3 \mathrm{se}$ muestra el espejo utilizado.

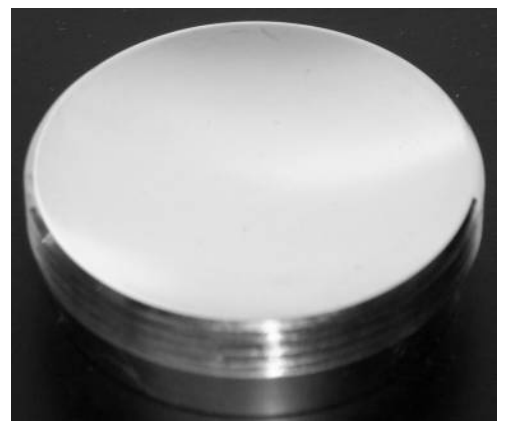

Figura 3. Foto del espejo de concavidad eliptica

Este sistema permite minimizar las aberraciones ópticas. Al estar compuesto el sistema por un espejo, la aberración cromática no está presente. La aberración esférica es reducida a través del uso de un perfil elíptico: el detector está ubicado en un foco de la elipse mientras que la imagen objetivo o fuente (piel de la frente) está en el otro foco, esto se ilustra en la Figura 4.

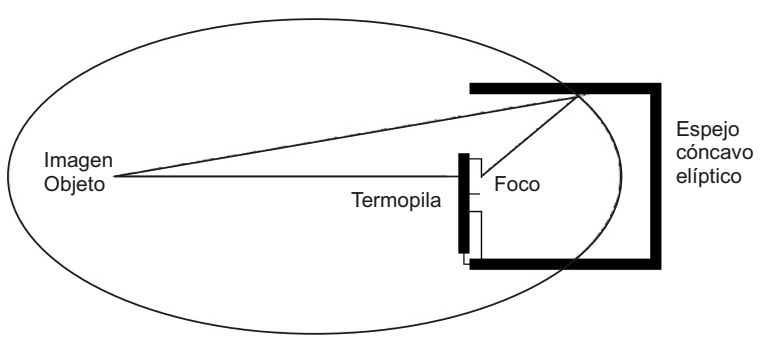

Figura 4. Esquema del sistema óptico

El sistema no requiere el uso de deflectores para reducir la radiación dispersa. La coma y el astigmatismo no están presentes estando los rayos principales en el eje. Cuando la distancia de enfoque varía entre 350 y $400 \mathrm{~mm}$, el área de la imagen objetivo se incrementa de $20 \mathrm{~mm} \times 20 \mathrm{~mm}$ hasta $25 \mathrm{~mm}$ x $25 \mathrm{~mm}$, lo que resulta aceptable para las aplicaciones mencionadas.

\subsection{Sistema mecánico}

El sistema mecánico está diseñado para soportar el espejo y el detector (sensor de radiación) del sistema óptico y para acomodarse fácilmente a las aplicaciones antes mencionadas. Este sistema consiste en un tubo cilíndrico, con la superficie interior roscada con un paso de $1.5 \mathrm{~mm}$, para reducir al máximo la radiación dispersa (luz ambiente del exterior que va hacia el detector). La estructura mecánica se muestra en la Figura 5. Las superficies externas e internas del tubo fueron ennegrecidas con anodizado negro mate. En las superficies externas se ubicaron cuatro agarraderas para permitir ensamblar el instrumento en otros equipos, como en camas de hospitales o en las puertas de inmigración de los aeropuertos, etc.

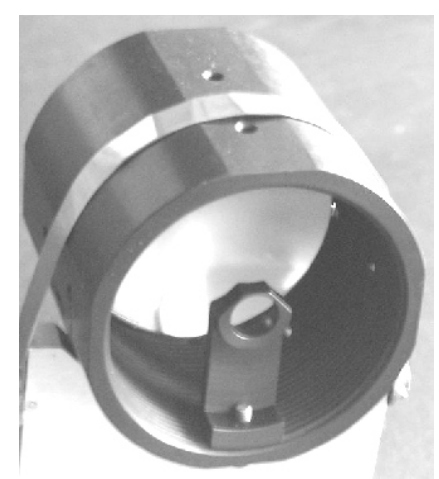

Figura 5. Sistema mecánico 


\subsection{Sensor de radiación}

Entre los distintos detectores que pueden ser utilizados en el rango de longitudes de onda deseado, las termopilas han sido escogidas debido a su buen desempeño $\left(\mathrm{D}^{*}=5 \times 10^{5}\right)$, bajo costo (pueden alcanzar US $\$ 5$ por pieza en producción en masa), facilidad de uso y porque ellas no requieren enfriamiento criogénico. Las únicas desventajas son: la necesidad de compensación por junturas frías y el tiempo de respuesta no muy corto, cercano a $0.3 \mathrm{~s}$, pero de todas formas adecuado para la mayoría de las aplicaciones mencionadas. De hecho, estos dispositivos entregan una señal influenciada no solo por la temperatura del objetivo, sino también por la temperatura ambiente (de la misma forma que las termocuplas), generalmente medida por un termistor insertado en el contenedor de la termopila. La compensación por junturas frías puede ser realizada durante la calibración del pirómetro, como se menciona en la sección 3.1.

Se probaron dos tipos de termopilas, de diferentes fabricante (Cal Sensor y Melexis). Las características de los dos dispositivos se reportan en la tabla 1, los dos sensores se muestran en la figura 6. La termopila Melexis es suministrada con un módulo para la compensación automática de la temperatura ambiente y entrega los valores de temperatura del objeto.

Tabla 1. Característica de las termopilas

\begin{tabular}{|lrr|}
\hline \multicolumn{1}{|c}{ Característica } & $\begin{array}{c}\text { Cal sensors mod. } \\
\text { TP-25 }\end{array}$ & $\begin{array}{l}\text { Melexis mod. } \\
\text { MLX90247D }\end{array}$ \\
\hline Área sensible & $4.00 \mathrm{~mm}^{2}$ & $4.81 \mathrm{~mm}^{2}$ \\
\hline FOV (Campo de vista) & $130^{\circ}$ & $100^{\circ}$ \\
\hline Material de la ventana & $\mathrm{CaF}_{2}$ & $\mathrm{CaF}_{2}$ \\
\hline Rango de longitud de onda & $0.2-11 \mu \mathrm{m}$ & $0.2-11 \mu \mathrm{m}$ \\
\hline Diámetro de apertura & $4.0 \mathrm{~mm}$ & $3.5 \mathrm{~mm}$ \\
\hline
\end{tabular}

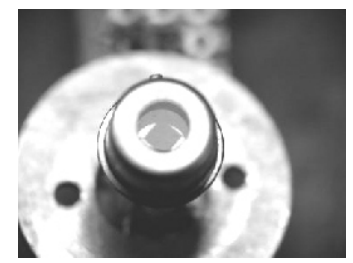

a

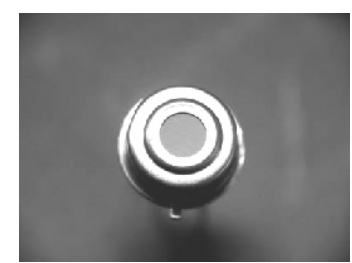

b
Figura 6. Foto de las dos termopilas utilizadas a. Cal Sensor; $b$ Melexis

\subsection{Caracterización de la termopila}

Se realizaron los estudios de caracterizacion de los dos tipos de termopilas, individualmente para reconocer la dependencia de la señal de la temperatura del objeto (calibración de la sensibilidad directa), y de la temperatura ambiente (calibración de la sensibilidad cruzada). Este metodo llamado cross sensitivity, mide la salida de la termopila en función de la juntura caliente, y consiste en apuntar la termopila a un cuerpo negro extendido con temperatura fija, mientras la temperatura ambiente va cambiando con la regulación sobre la planta calefactora del laboratorio o sistema de aire acondicionado. De esta forma, se obtiene un cambio continuo en la salida del sistema de amplificacion de la termopila que mide la temperatura (ST) del objeto (medida en voltios) versus la temperatura del ambiente, a través de la medida de resistencia del termistor para cada temperatura fijada en el cuerpo negro (TBB).

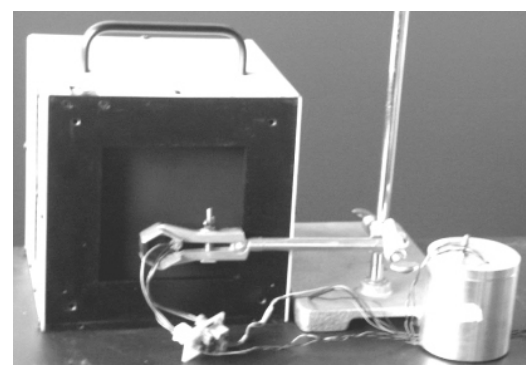

Figura 7. Calibración de la termopila con amplificador operacional, de frente al cuerpo negro (black body)

En la figura $8 \mathrm{a}$ y 8 b se muestran los resultados de esta calibración, correspondiente al valor de la medida de la termopila en voltios y diferentes temperaturas, a través de la resistencia del termistor, en ohmios, mostrada en la abscisa. Esto se realizó después de salir del sistema de amplificación con OPAM, figura 7.

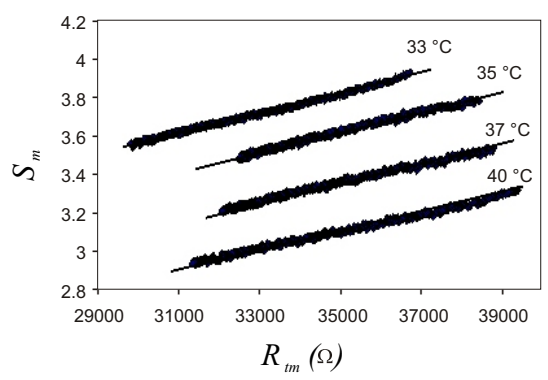

a 


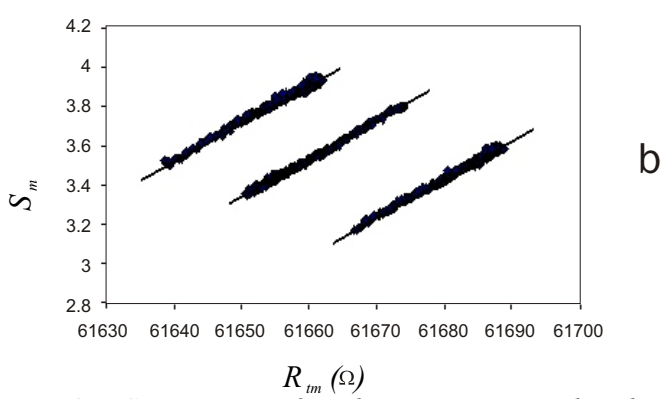

Figura 8. Caracterización directa y cruzada de las termopilas Cal sensors (a) y Melexis (b). Comportamiento de la señal de salida ST en voltios versus la temperatura ambiente, dada por la resistencia del termistor, RTM en Ohmios y la temperatura del cuerpo negro en ${ }^{\circ} \mathrm{C}$.

Se escoge la termopila Melexis, ya que esta casa productora suministra un circuito integrado (chip) con termopila y sistema de compensación (corrección del efecto de la temperatura ambiente), pero con programación de fabrica, el $M L X 90601 B$ (figura 9, PCB izquierdo ) y otro un chip programable, el $M L X 90601 K Z A-B K A$ (figura 9, chip derecho ); siendo este último utilizado para el desarrollo en el prototipo final del pirómetro.

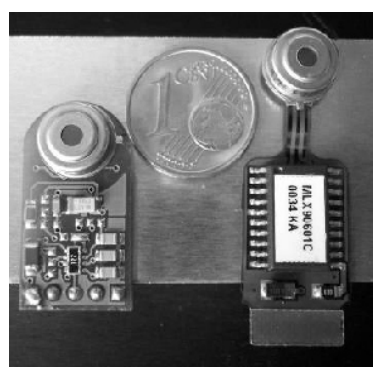

Figura 9. Dispositivos Melexis experimentadas en el pirómetro, de derecha a izquierda: $P C B \quad M L X 90601 B$ y chip programable MLX $90601 K Z A-B K A$

\section{Resultados y discusión}

\subsection{Caracterización del pirómetro}

Cuando se inserta en el instrumento, la termopila es influenciada por la temperatura ambiente, no solo por la temperatura de junturas frías, sino también por otras dos razones. Primero, porque el campo de vista (FOV) es más amplio que el ángulo subtendido desde el detector hacia el espejo, y segundo porque la reflectividad del espejo es muy alta $(0.95$ a 0.98$)$ debido al recubrimiento de oro, $y$ puede degradarse por contaminación por polvo y oxidación. Como resultado, estos tres fenómenos con la temperatura ambiente, y pueden ser tomados en cuenta por una caracterización general. Una vez más, esta caracterización se realiza cambiando ambas, la temperatura ambiente y la temperatura de la fuente (cuerpo negro), donde el pirómetro es apuntado. La figura 10 muestra el resultado de estas pruebas. En el eje de las abscisas se reporta la temperatura del cuerpo negro, y las distintas curvas se relacionan con diferentes temperaturas ambiente. Se observa un comportamiento parabólico de la señal contra la temperatura del cuerpo negro, mientras que se presenta un comportamiento lineal contra la temperatura ambiente.

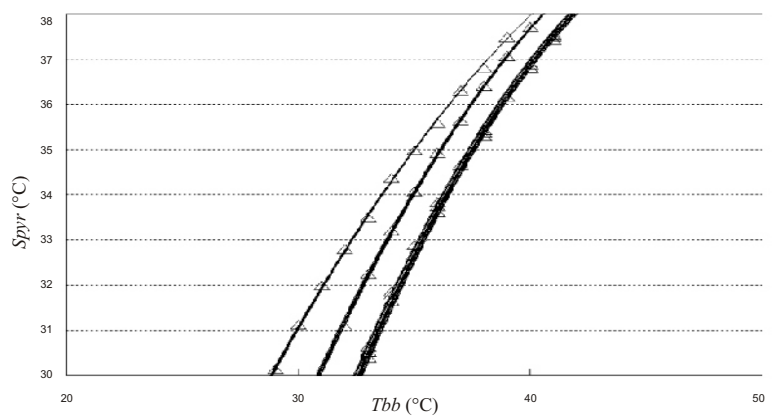

Figura 10: Caraterización del pirómetro. Datos registrados del pirómetro vs temperatura ambiente y temperatura del cuerpo negro, y líneas de regresión.

El procesamiento de estos datos es posible por medio de una regresión lineal múltiple entre la variable dependiente, la señal del pirómetro Spyr, y las dos variables independientes: la temperatura del cuerpo negro $t b b$ y la resistencia del termistor, Rtm, en función de la temperatura ambiente. El modelo de regresión está descrito por la siguiente ecuación:

$$
S_{p y r}=b_{1}+b_{2} \cdot t_{b b}+b_{3} \cdot R_{t m}+b_{4} \cdot t_{b b}{ }^{2}+b_{5} \cdot t_{b b} R_{t m}+b_{6} \cdot t_{b b}{ }^{2} R_{t m}
$$

Tabla 2. Resultado de la regresión de mínimos cuadrados de los datos obtenidos, usando la ecuación (1) como modelo

\begin{tabular}{|lrr|}
\hline Parámetro & Valor & $\begin{array}{c}\text { Incertidumbre } \\
\text { estándar }\end{array}$ \\
\hline$b_{1}$ & 128,2 & 5,4 \\
\hline$b_{2}$ & $-3,30$ & 0,4 \\
\hline$b_{3}$ & $-7,9$ & 0,4 \\
\hline$b_{4}$ & $-0,03$ & 0,002 \\
\hline$b_{5}$ & 0,43 & 0,02 \\
\hline$b_{6}$ & $-6,2 \cdot 10^{-3}$ & 0,8 \\
\hline
\end{tabular}


La mejor aproximación de los coeficientes se muestra en la tabla 2 con sus valores de incertidumbres estándar (Brandt, 1970); los comportamientos relativos se muestran en la figura 10. El error total de predicción del algoritmo de mínimos cuadrados es de $0.081 \mathrm{~V}$.

Para obtener la temperatura de la frente de una persona, se debe despejar el término correspondiente a la temperatura del cuerpo negro (temperatura real medida por el pirómetro) de la ecuación 1 , para dar:

$t_{b b}=\frac{-b_{2}-b_{5} R_{t m}-\sqrt{\left(b_{2}+b_{5} R_{t m}\right)^{2}-4\left(b_{4}+b_{6} R_{t m}\right)\left(b_{1}+b_{3} R_{t m}-S_{p y r}\right)}}{2\left(b_{4}+b_{6} R_{t m}\right)}$

El error estándar de estos coeficientes, obtenidos del error de predicción dados por la regresión de la ecuación 1, a través de la ley de propagación del error, es aproximadamente de $0.15^{\circ} \mathrm{C}$. Este valor puede verse como la incertidumbre de calibración de la temperatura evaluada, incertidumbre tipo B, según GUM, ISO (1995).

\subsection{Pruebas realizadas con el pirómetro}

Una serie de pruebas han sido efectuadas apuntando el pirómetro en la frente de varias personas, y cambiando la distancia de trabajo de $350 \mathrm{~mm}$ a $400 \mathrm{~mm}$. Todos los valores detectados estuvieron en el rango entre $34^{\circ} \mathrm{C}$ y $34.5^{\circ} \mathrm{C}$, confirmando que la temperatura de la frente es casi constante entre diferentes personas, y depende solo de factores ambientales (i.e. temperatura del aire, velocidad del aire, temperatura media radiante, humedad relativa) de acuerdo con la teoría Fanger del confort humano (Fanger, 1973).

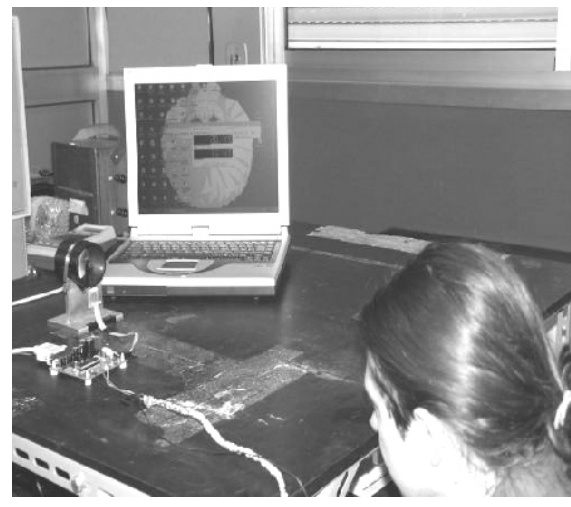

a.

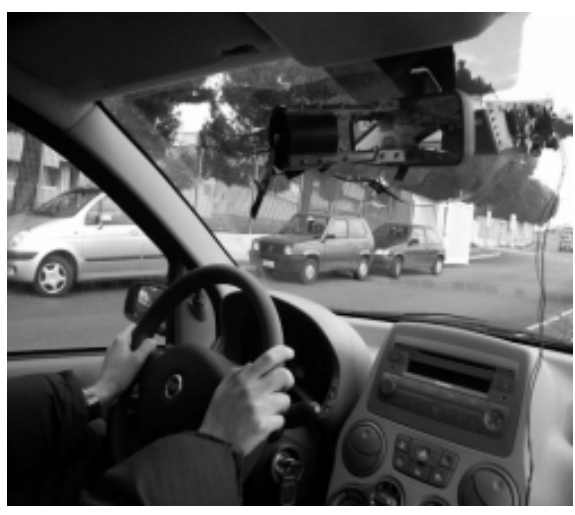

b.

Figura 11. Pruebas típicas de medición de la temperatura de la frente: a. Laboratorio b. Interior de un vehiculo (Fiat Panda 2003)

La figura 11 muestra el escenario de dos pruebas típicas realizadas. Otra de las pruebas realizadas consistió en observar posibles cambios de la medición de la temperatura de la frente con el instrumento ante disturbios de tipo eletromagnético, y no se encontraron problemas.

\subsection{Precisión del instrumento}

La regresión de mínimos cuadrados entrega un valor de incertidumbre de $0.15^{\circ} \mathrm{C}$ (incertidumbre de calibración). En medidas repetidas se dieron resultados en un rango de $\pm 0.1^{\circ} \mathrm{C}$, entonces se puede atribuir una incertidumbre estándar de 0.05 , como incertidumbre tipo A (ISO, 1995). Otras fuentes de incertidumbre (tipo B) están asociadas a:

Efecto de la temperatura ambiente: en la calibración se asumió que la temperatura de la termopila es la misma temperatura del espejo y del cuerpo del pirómetro. Se puede asumir esto, debido al pequeño tamaño de los instrumentos, y al material (aluminio). De cualquier forma puede estimarse una diferencia máxima de $0.1^{\circ} \mathrm{C}$ entre los diferentes componentes del instrumento, esta diferencia contribuye en una cantidad despreciable a la incertidumbre total.

Efecto de la luz ambiente: se notó un efecto despreciable debido a la presencia e intensidad de luz de día o a la iluminación artificial durante la noche. 
Contaminación del espejo: se realizaron pruebas de laboratorio con el espejo sin protección. Después de un año el espejo se volvió un poco opaco y fue necesaria su limpieza. Una protección con una capa delgada de $\mathrm{SiO}$ o $\mathrm{MgO}$ debería reducir el problema, y permitir una limpieza segura y más fácil.

Finalmente, una incertidumbre estándar de $0.19^{\circ} \mathrm{C}$ puede atribuirse al instrumento.

\section{Conclusiones y recomendaciones}

El instrumento obtenido mide la temperatura de la frente de una persona con una precisión mayor que $0.2^{\circ} \mathrm{C}$. Es no invasivo (sin contacto), con una distancia de trabajo de entre 350 y $400 \mathrm{~mm}$.

Los problemas de contaminación en el espejo por polvo y polución, que pueden causar un deterioro del espejo, se pueden disminuir con una deposición en vacío de una capa fina de $\mathrm{SiO}$ o $\mathrm{MgO}$ en el espejo. Si la distancia de trabajo del pirómetro debe cambiarse para satisfacer otros requerimientos, se debe diseñar un nuevo sistema óptico similar al mostrado en la figura 4.

El prototipo elaborado del instrumento es liviano (solo $0.2 \mathrm{~kg}$ de peso), fácil de usar y práctico en aplicaciones como el monitoreo de la temperatura de pacientes en cama o para la medición de la temperatura de viajeros que cruzan a través de puertas de aeropuertos. De esta manera, se puede determinar cuándo una persona tiene fiebre, y se puede prevenir la contaminación y diseminación de virus y enfermedades peligrosas, como el ebola, la fiebre aviar, etc. Otras aplicaciones se pueden encontrar si los requerimientos son satisfechos por las características del instrumento: i.e., distancia de trabajo entre 350 y $400 \mathrm{~mm}$, área del objetivo desde $20 \mathrm{~mm} \times 20 \mathrm{~mm}$ hasta $25 \mathrm{~mm} \times 25 \mathrm{~mm}$, rango de temperatura entre 30 y $45{ }^{\circ} \mathrm{C}$. Si la emisividad es diferente de la unidad (cuerpo negro), su valor debe ser conocido en el rango de longitudes de onda usadas para la medición (i.e., 1 a $12 \mu \mathrm{m}$ ), para corregir las medidas de forma apropiada.

\section{Agradecimientos}

Este proyecto fue financiado por el Centro de Investigaciones FIAT, Roma Italia, y desarrollado en el Laboratorio de Mediciones Térmicas y Transferencia de Calor de la Facultad de Ingeniería de la Universidad de Roma Tor Vergata. El instrumento desarrollado fue registrado bajo Patente \# RM2004A000528 Pirometro ad infrarossi per la misura della temperatura della pelle.

\section{Referencias bibliográficas}

Brandt, S. (1970). Statistical and computational methods in data analysis. Amsterdam: North Holland Pub.

Fanger, P.O. (1973). Thermal comfort. New York: McGraw Hill Book Company.

ISO. (1995). Guide to the Expression of Uncertainty in Measurement (GUM).

Kataoka, H., Kano, H., Yoshida, H., Saijo, A., Yasuda, M., \& Osudi, M. (1998). Development of a skin temperature measuring system for noncontact stress evaluation. Proceedings of the 20th International Annual International Conference of the IEEE Engineering in Medicine and Biology Society 20 (2), 940-943.

Lee, H. M., Cho, C. K., Yun, M. H., \& Lee, M. W. (1998). Development of a temperature control procedure for a room air-conditioner using the concept of just noticeable difference (JND) in thermal sensation. Industrial Ergonomics 22 (3), 207-216.

Taniguchi, Y., Aoki, H., Fujikake, K., Tanaka, H. \& Kitada, M. (1992). Study on Car Air Conditioning System Controlled by Car Occupants' Skin Temperature Part 1: Research on Methods of Quantitative Evaluation of Car Occupants' Thermal Sensation by Skin Temperatures, SAE Paper No. 920169. 